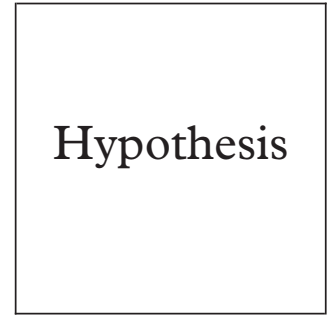

\section{HIV protease inhibitors, the lipodystrophy syndrome and polycystic ovary syndrome-is there a link?}

\author{
J D Wilson, R J C Dunham, A H Balen
}

\section{Department of} Genitourinary

Medicine, The General Infirmary at Leeds, Leeds LS1 3EX

J D Wilson

\section{Department of} Radiology, Wharfedale General Hospital, Otley, LS21 2LY

R J C Dunham

Reproductive Medicine Unit, The General Infirmary at Leeds, Leeds LS1 3EX

A H Balen

Correspondence to: Dr Janet D Wilson, Department of

Genitourinary Medicine,

The General Infirmary at Leeds, Great George Street, Leeds LS1 3EX.

Accepted for publication 18 June 1999
A metabolic syndrome of insulin resistance, dyslipidaemia, central/visceral obesity, and peripheral lipodystrophy has been described with HIV protease inhibitors (PIs). ${ }^{1-3}$ A recent clinical presentation has led us to hypothesise that PIs can precipitate the metabolic changes associated with polycystic ovary syndrome (PCOS) by causing insulin resistance, and that people developing the metabolic/lipodystrophy syndrome may have genetic predisposition to PCOS.

In February 1997, a 37 year old woman presented with newly diagnosed HIV-1 infection, and a CD 4 count of 80 cells $\times 10^{6} / 1$. She began antiretroviral therapy with zidovudine, lamivudine, and saquinavir $600 \mathrm{mg}$ thrice daily. She had a good response to treatment with a rise in CD4 count to 330 cells $\times 10^{6} / 1$ and a viral load $<400$ copies/ml, but found adherence to a three times daily regimen difficult. So in March 1998 her PI was changed to ritonavir $400 \mathrm{mg}$ plus saquinavir $400 \mathrm{mg}$ allowing twice daily dosing. In June 1998 she noticed dark hairs on her chin, upper lip, and cheeks, increasing abdominal girth, and a fat pad at the back of her neck. Her weight was stable at $57 \mathrm{~kg}$. There were no recent changes in her menstrual cycle, which had a tendency to be erratic. She was nulliparous. Examination confirmed central obesity, a buffalo hump, and facial hirsutism. Her plasma follicle stimulating hormone (4.7 $\mathrm{IU} / 1$; normal range $<8 \mathrm{IU} / \mathrm{l}$ ) and luteinising hormone (22.3 IU/1; normal range <10 IU/1) were consistent with PCOS. Total serum testosterone level was normal $(0.8 \mathrm{nmol} / \mathrm{l}$; normal range $0.0-2.8 \mathrm{nmol} / \mathrm{l}$ ), as were thyroid function tests, cortisol, and prolactin. Transvaginal pelvic ultrasound confirmed polycystic ovaries. Her fasting triglyceride $(2.7 \mathrm{mmol} / \mathrm{l})$ and cholesterol $(7.4 \mathrm{mmol} / \mathrm{l})$ levels were raised but blood glucose was normal. She chose electrolysis to control her hirsutism. So far she has decided to stay on the same antiretroviral treatment as she feels the side effects have not outweighed the clinical benefits.

The pathogenesis of PCOS is unknown but there is evidence of genetic susceptibility, possibly with autosomal dominant transmission with variable penetrance. ${ }^{45} \mathrm{Up}$ to $20 \%$ of women may have the morphological appearance of polycystic ovaries on ultrasound. ${ }^{6}$ Women with PCOS are more likely to have hyperinsulinaemia and insulin resistance than weight matched controls, ${ }^{5}$ with decreased insulin sensitivity in peripheral tissues, particularly muscle and adipose tissue, but not in liver or ovary. The exact mechanism of insulin resistance is unclear but an intrinsic post-receptor defect has been identified. ${ }^{5}$ They are also more likely to have central/visceral obesity, with a significantly higher waist/hip ratio than age and weight matched controls. ${ }^{7}$ Obesity causes a more pronounced insulin resistance in women with PCOS than in controls. ${ }^{5}$ The hyperinsulinaemia stimulates production of ovarian androgens but inhibits synthesis of sex hormone binding globulin, resulting in increased free testosterone levels. It is the free testosterone that is biologically active. This may be elevated even when total testosterone is normal. ${ }^{68}$ The biochemical basis of the hyperandrogenaemia is an intrinsic abnormality of cytochrome $\mathrm{P} 450 \mathrm{c} 17 \alpha$ which is the rate limiting enzyme in ovarian androgen biosynthesis. ${ }^{5}$ It is still unclear whether the genetic predisposition to insulin resistance and ovarian androgen production represents one genetic defect or several. ${ }^{5}$ Other associated metabolic abnormalities include dyslipidaemia and non-insulin dependent diabetes, and possibly an increased risk of cardiovascular disease ${ }^{6}{ }^{8}$ Full expression of PCOS probably requires the underlying genetic disorder plus an abnormality of insulin metabolism-for example, non-obese women may develop symptoms of PCOS with weight gain. This interrelation may explain the wide spectrum of clinical and metabolic manifestations. ${ }^{68}$

This patient had underlying menstrual problems and ovarian morphology confirming the diagnosis of PCOS. The increase in her symptoms appears to have been precipitated by the two PIs. An increased rate of lipodystrophy and higher insulin levels have been reported with a ritonavir/saquinavir combination. ${ }^{2}$ Insulin resistance has been reported with all the PIs, ${ }^{1}$ and this could have triggered the androgenaemia in this genetically susceptible woman. The PIs are not the first drugs to be implicated in precipitating the manifestations of PCOS in susceptible women; this has also been recognised as a possible problem with sodium valproate. ${ }^{9}$

Many of the metabolic manifestations of PCOS are similar to those of the lipodystrophy syndrome with PIs. Our patient would have been diagnosed as having lipodystrophy not PCOS were it not for the hirsutism. Male relatives of women with PCOS have been found to have insulin resistance, and a high frequency of non-insulin dependent diabetes and dyslipidaemia. ${ }^{5}$ Although the exact clinical, 
hormonal, and metabolic manifestations of PCOS in males are unknown, the genetic predisposition may be expressed as premature balding. ${ }^{4}$ If our hypothesis is correct such balding males, and females with polycystic ovaries, should be more likely to develop lipodystrophy syndrome, hyperlipidaemia, and diabetes mellitus with PIs.

Potential causes of the metabolic abnormalities and lipodystrophy with PIs have been suggested. ${ }^{10}{ }^{11}$ Insulin resistance, hypertriglyceridaemia, and central obesity are interrelated so it is difficult to know which occurs first. Evidence is mounting that the initial factor is development of insulin resistance, which can lead to the other metabolic manifestations. ${ }^{11}$ This is probably the mechanism in those with mild lipodystrophy, which has been reported in up to $83 \%$ of people taking PIs. ${ }^{12}$ However, we suggest that development of the full metabolic disorder (which has been reported in $11 \%{ }^{12}$ ) occurs in those with a genetic predisposition to insulin resistance (in the form of PCOS) when triggered by the additional insulin resistance caused by the PIs. Supporting this is the correlation of severity of lipodystrophy with insulin resistance, ${ }^{12}$ and the hyperandrogenaemia found in women with PI lipodystrophy. ${ }^{13}$ Women with hyperinsulinaemia but without PCOS do not get raised androgen levels. ${ }^{5}$ The improvement of the PI associated metabolic changes with metformin further supports insulin resistance as the primary problem. ${ }^{14}$ Similar responses to metformin have been described in women with PCOS, with reduction in insulin, lipoproteins, plasminogen activator inhibitor 1, central obesity, ovarian cytochrome P450c17 $\alpha$ activity, and hence testosterone production. ${ }^{8}$ The reduction in testosterone following metformin for lipodystrophy again supports a linked abnormality of insulin action and androgen production in these individuals, as without this testosterone levels would not be expected to change. ${ }^{14}$ Lipodystrophy syndrome has been described in HIV disease without PIs. Our hypothesis supports this as any cause of increased insulin resistance-for example, other drugs, could precipitate the changes in the genetically susceptible. This genetic predisposition would explain why many people taking PIs have no metabolic disorder, and why there has been such a wide spectrum of clinical and metabolic manifestations described similar to the heterogeneity of PCOS.

1 Miller KD, Jones E, Yanovski JA, et al. Visceral abdominalfat accumulation associated with use of indinavir. Lancet 1998;351:871-5.

2 Carr A, Samaras K, Burton S, et al. A syndrome of peripheral lipodystrophy, hyperlipidaemia and insulin resistance in patients receiving HIV protease inhibitors. AIDS in patients reciv

3 Walli R, Herfort O, Michl GM, et al. Treatment with protease inhibitors associated with peripheral insulin resistance and impaired oral glucose tolerance in HIV-1 infected patients. AIDS 1998;12:F167-73.

4 Franks S, Gharani N, Waterworth D, et al. The genetic basis of polycystic ovary syndrome. Human Reprod 1997;12 2641-8.

5 Dunaif A. Insulin resistance and the polycystic ovarian syndrome: mechanisms and implications for pathogenesis. Endocr Rev 1997;18:774-800.

6 Franks S. Polycystic ovary syndrome. $N$ Engl f Med 1995;333:853-61.

7 Douchi T, IjuinH, Nakamura S, et al. Body fat distribution in women with polycystic ovary syndrome. Obstet Gynecol 1995;86:516-9.

8 Hopkinson ZEC, Satter N, Fleming R, et al. Polycystic ovarian syndrome: the metabolic syndrome comes to gynaecology. BMF 1998;317:329-32.

9 Isojarvi JIT, Laatikainen TJ, Parakinen AJ, et al. Polycystic ovaries and hyperandrogenism in women taking valproate for epilepsy. N Engl f Med 1993;329:1383-8.

10 Carr A, Samaras K, Chisholm DJ, et al. Pathogenesis of HIV-1-protease inhibitor associated peripheral lipodystrophy, hyperlipidaemia, and insulin resistance. Lancet 1998; 352:1881-3.

11 Martinez E, Gatell J. Metabolic abnormalities and use of HIV-1 protease inhibitors. Lancet 1998;352:821-2.

12 Carr A, Samaras K, Thorisdottir A, et al. Diagnosis and prediction of HIV protease inhibitor induced lipodystrophy and impaired glucose tolerance. Sixth Conference on Retroviruses and Opportunistic Infections, Chicago, February 1999 (abstract 641)

13 Saint-Marc T, Touraine JL. Truncal adiposity syndrome, insulin resistance, endocrine and metabolic disorders in women receiving HIV protease inhibitors. AIDS 1998; 12(suppl 4):S53.

14 Saint-Marc T, Touraine JL. Effects of metformin on insulin resistance and central adiposity in patients receiving effective protease inhibitor therapy. Sixth Conference on Retroviruses and Opportunistic Infections, Chicago, February 1999 (abstract 672). 